\title{
Efeito da classificação dos ovos sobre o rendimento de incubação e os pesos do pinto e do saco vitelino
}

[Effect of egg classification prior to setting on hatchability, embryonic mortality and chick and yolk sac weights]

\author{
J.S.R. Rocha $a^{1}$, L.J.C. Lara $^{2}$, N.C. Baião ${ }^{2 *}$, S.V. Cançado ${ }^{2}$, L.E.C. Baião $o^{3}$, T.R. Silva ${ }^{3}$ \\ ${ }^{1}$ Aluna de pós-graduação - EV-UFMG - Belo Horizonte, MG \\ ${ }^{2}$ Escola de Veterinária - UFMG \\ Caixa Postal 567 \\ 30123-970 - Belo Horizonte, MG \\ ${ }^{3}$ Asa Alimentos - Brasília, DF
}

\begin{abstract}
RESUMO
Dois experimentos foram realizados para avaliar, no primeiro, o efeito da idade da matriz sobre a uniformidade do peso de 1080 ovos e gemas e, no segundo, a influência da classificação de 9260 ovos sobre a eclodibilidade, a mortalidade embrionária e os pesos, absoluto e relativo, do pinto e do saco vitelino. Os tratamentos do experimento I foram ovos das matrizes com 31 (M31), 38 (M38) e 43 (M43) semanas de idade. No segundo, os ovos das matrizes com 31, 38 e 43 semanas foram distribuídos em seis tratamentos; A: ovos com pesos entre 66 e 72g, 33,3\% deles originados de matrizes com diferentes idades; B: ovos com pesos entre 58 e $65 \mathrm{~g}, 33,3 \%$ deles originados de matrizes com diferentes idades; C: ovos com pesos entre 52 e $57 \mathrm{~g}, 33,3 \%$ deles originados de matrizes com diferentes idades; D: ovos com pesos entre 52 e $72 \mathrm{~g}$, produzidos por matrizes com 31 semanas; E: ovos com pesos entre 52 e $72 \mathrm{~g}$, produzidos por matrizes com 38 semanas; e F: ovos com pesos entre 52 e $72 \mathrm{~g}$, produzidos por matrizes com 43 semanas. As aves de M31 produziram ovos com pesos do ovo e da gema mais uniformes que as do M43. Ovos do tratamento A apresentaram a pior eclosão e a maior mortalidade embrionária após 15 dias de incubação, e produziram pintos com maiores pesos absoluto e relativo do saco vitelino.
\end{abstract}

Palavras-chave: pinto, classificação de ovos, eclosão, mortalidade embrionária, saco vitelino

\begin{abstract}
Two experiments were carried out to study the effects of broiler breeder age on weight uniformity of 1,080 eggs and yolks, and the effects of 9,216 eggs classified prior to setting on hatchability, embryonic mortality, and the relationship between chick and yolk sac weights. In the first study, the three treatments were eggs from broiler breeders on $31^{\text {st }}$ (M31), $38^{\text {th }}$ (M38), and 43 ${ }^{\text {th }}$ (M43)-week of age. In the second study, eggs produced by three broiler breeders flocks $\left(31^{\text {st }}, 38^{\text {th }}\right.$, and $43^{\text {th }}$ wh of age) were divided in six treatments; A: eggs weighting from 66 to $72 \mathrm{~g}, 33,3 \%$ of them produced by broiler breeders of different ages; $B$ : eggs weighting from 58 to $65 \mathrm{~g}, 33,3 \%$ of them produced by broiler breeders of different ages; $C$ : eggs weighting from 52 to $57 \mathrm{~g}$, 33,3\% of them produced by broiler breeders of different ages; D: eggs from 31-wk-old broiler breeders, weighting from 52 to 72g; E: eggs from 38-wk-old broiler breeders, weighting from 52 to 72g; F: eggs from 43-wk- old broiler breeders weighting from 52 to $72 \mathrm{~g}$. Eggs produced by M31 had egg and yolk weights more uniform than the eggs collected from M43. Treatment A had the worst hatchability, higher embryonic mortality after 15 days of incubation, and produced heavier hatched chicks with superior absolute and relative weights of yolk sac.
\end{abstract}

Keywords: broiler chick, egg classification, hatchability, embryonic mortality, yolk sac

Recebido em 2 de outubro de 2007

Aceito em 9 de julho de 2008

*Autor para correspondência (corresponding author)

E-mail: baiao@vet.ufmg.br

Apoio: FAPEMIG 


\section{INTRODUÇÃO}

As matrizes pesadas produzem ovos menores no início do período de produção, os quais apresentam baixo rendimento de incubação. Esse fato tem sido relacionado às características dos ovos de matrizes jovens, pois, quando comparados aos ovos de galinhas mais velhas, apresentam a casca mais espessa e o albúmen mais denso, levando a uma redução da perda de umidade e das trocas gasosas (Brake et al., 1997). Esses fatores, associados à menor disponibilidade de nutrientes para o embrião, devido à baixa capacidade das aves jovens de transferir lipídios para a gema do ovo, podem comprometer a viabilidade embrionária nos estádios iniciais de desenvolvimento e reduzir a eclosão (Benton e Brake, 1996; Fasenko, 2003).

Tona et al. (2001) avaliaram o rendimento de incubação de um lote de matrizes Cobb desde 27 até 60 semanas de idade e observaram a menor mortalidade embrionária e, conseqüentemente, a melhor eclodibilidade nos ovos provenientes de matrizes com 40 a 42 semanas de idade.

Em matrizes com idade avançada, ocorre aumento no peso do ovo com redução na eclodibilidade, pois os embriões desenvolvidos nos ovos maiores são menos tolerantes ao excessivo calor metabólico produzido no final do período de incubação (French, 1997; Lourens et al., 2006).

Uma vez que os efeitos da idade da matriz sobre o peso do ovo e os rendimentos de incubação são conhecidos, os incubatórios deveriam classificar os ovos, antes da incubação, considerando apenas a idade da matriz. Entretanto, após a primeira metade do período de incubação, a correlação entre peso do ovo e peso do embrião aumenta, chegando a atingir valores entre 0,5 a 0,95 no momento da eclosão (Wilson, 1991). Essa influência é bem conhecida, e os trabalhos atuais demonstram que $\mathrm{o}$ peso do pinto representa entre 66 e $71 \%$ do peso do ovo (Joseph e Moran Jr., 2005a; Michalsky et al., 2005; Fiúza et al., 2006; Marinho et al., 2006; Pappas et al., 2006). Baseado nesses aspectos, os incubatórios adotam um sistema de classificação dos ovos pelo peso, desconsiderando a idade da matriz. Esse procedimento visa atender à demanda do cliente por um pinto mais pesado e com peso mais uniforme.
$\mathrm{O}$ rendimento de incubação e o peso e a qualidade do pinto dependem de vários fatores que incluem, entre outros, a idade da matriz que, por sua vez, influencia o peso do ovo. Esses efeitos têm sido estudados separadamente e estão bem documentados na literatura científica conforme descrito anteriormente. Entretanto, até o momento, nenhuma dessas pesquisas com incubação considerou o sistema de classificação dos ovos pelo peso que, apesar de não ser recomendado, é amplamente adotado pelos incubatórios. Assim, os objetivos neste trabalho foram verificar, no experimento I, a influência da idade da matriz sobre a uniformidade do peso do ovo e da gema e, no experimento II, os efeitos da classificação dos ovos no incubatório sobre o rendimento de incubação e os pesos absoluto e relativo do pinto e do saco vitelino.

\section{MATERIAL E MÉTODOS}

Nos dois experimentos foram utilizados ovos incubáveis de matrizes pesadas da linhagem Cobb, produzidos por três lotes com idades de 31,38 e 43 semanas. Todos os ovos foram produzidos no mesmo dia e coletados às 9 horas, correspondendo à segunda coleta do dia.

No experimento I, uma amostra aleatória de 360 ovos de cada idade das matrizes, 31, 38 e 43 semanas, totalizando 1080 ovos, foram pesados individualmente, no mesmo dia da postura, para avaliar a uniformidade do peso. Para as avaliações da uniformidade dos pesos das gemas e das proporções de gema, casca e albúmen em relação ao peso do ovo, foram quebrados 180 ovos de cada idade de matriz (anteriormente pesados). As gemas, separadas manualmente, foram pesadas individualmente. As cascas lavadas em água corrente para a retirada dos resíduos do albúmen foram secas em temperatura ambiente por 24 horas antes da pesagem individual. $\mathrm{O}$ peso do albúmen foi obtido pela diferença entre os pesos do ovo inteiro e da gema e da casca. Os tratamentos, definidos pelas idades das matrizes, foram os seguintes: M31, ovos de matrizes com 31 semanas de idade; M38, ovos de matrizes com 38 semanas de idade; e M43, ovos de matrizes com 43 semanas de idade. $O$ delineamento experimental foi inteiramente ao acaso, constituído por três tratamentos, com 360 repetições para avaliar a uniformidade do peso dos ovos e 180 repetições para avaliar a uniformidade do peso da gema e as 
porcentagens de gema, albúmen e casca. As variâncias dos pesos dos ovos e das gemas foram comparadas pelo teste de Fisher, e as médias dos pesos relativos dos componentes foram comparadas pelo teste SNK, segundo Sampaio (2002), utilizando o programa SAEG (Sistema..., 2005).

No experimento II, foram utilizados 9216 ovos incubáveis dos três lotes de matrizes para constituírem seis tratamentos. Os tratamentos, definidos pela classificação dos ovos no incubatório, foram A: ovos com pesos entre $66 \mathrm{e}$ $72 \mathrm{~g}, 33,3 \%$ deles originados de matrizes com diferentes idades; B: ovos com pesos entre 58 e $65 \mathrm{~g}, 33,3 \%$ deles originados de matrizes com diferentes idades; C: ovos com pesos entre 52 e $57 \mathrm{~g}, 33,3 \%$ deles originados de matrizes com diferentes idades; D: ovos com pesos entre 52 e $72 \mathrm{~g}$, produzidos por matrizes com 31 semanas; $\mathrm{E}$ : ovos com pesos entre 52 e $72 \mathrm{~g}$, produzidos por matrizes com 38 semanas; e F: ovos com pesos entre 52 e $72 \mathrm{~g}$, produzidos por matrizes com 43 semanas. Os tratamentos A, B e C simularam a classificação que utiliza o peso como único critério. Estes foram constituídos igualmente pelas três idades de matrizes para evitar que o efeito de uma idade de matriz influenciasse as respostas obtidas. Os tratamentos D, E e F representaram a classificação mais adequada dos ovos para incubação. Os ovos, colocados em bandejas próprias para incubação com capacidade para 96 ovos cada e identificadas de acordo com os tratamentos, foram armazenados por dois dias, sob temperatura de $19^{\circ} \mathrm{C}$ e umidade relativa de $75 \%$.

Antes da incubação, três ovos de cada bandeja foram pesados, individualmente, e identificados a lápis. Como cada bandeja dos tratamentos $\mathrm{A}, \mathrm{B}$ e C continha 32 ovos de cada idade de matriz, tomou-se cuidado para que um ovo de cada idade de matriz por bandeja fosse pesado e identificado.

Aos 10 dias de incubação, foi realizada uma ovoscopia de todos os 9216 ovos, para retirar os inférteis ou com mortalidade embrionária precoce.

A transferência dos ovos da incubadora para o nascedouro foi feita com 456 horas de incubação (19 dias), e todos os embriões foram vacinados in ovo contra a doença de Marek. Antes da colocação das bandejas no nascedouro, os três ovos de cada bandeja, previamente identificados, foram envolvidos por redes de náilon individuais. Esse procedimento foi adotado para permitir que a identificação dos pintos fosse a mesma da casca do ovo no momento da eclosão.

A retirada dos pintos do nascedouro ocorreu com 504 horas (21 dias) de incubação. Imediatamente após o nascimento, os pintos foram levados à sala de pintos, com temperatura média de $25^{\circ} \mathrm{C}$ e umidade relativa média de $75 \%$, para contagem. O número de ovos não eclodidos de cada bandeja foi também registrado. Os ovos não eclodidos foram quebrados e examinados para determinar a fase em que ocorreu a mortalidade embrionária. Os pintos que nasceram dentro das redes foram abatidos para pesagem individual deles e dos respectivos sacos vitelinos.

As variáveis estudadas foram: taxas de eclosão em relação ao número total de ovos incubados $\mathrm{e}$ ao número de ovos férteis (avaliado na ovoscopia), taxa de mortalidade embrionária embriões que morreram entre zero e sete dias de incubação, entre oito e 14 dias, entre 15 e 18 dias, e entre 19 e 21 dias mais os ovos bicados com embriões vivos e mortos, e ovos contaminados e desidratados -, pesos absoluto e relativo do pinto $\mathrm{e}$ do saco vitelino. $\mathrm{O}$ delineamento experimental foi inteiramente ao acaso, constituído por seis tratamentos. Para a avaliação do rendimento de incubação, eclodibilidade e mortalidade embrionária, cada bandeja com 96 ovos foi considerada uma repetição, sendo 16 repetições por tratamento. Para a avaliação dos pesos absoluto e relativo do pinto e do saco vitelino, cada pinto foi considerado uma repetição; portanto, foram utilizadas 48 e 32 repetições por tratamento, respectivamente. A diferença entre as médias foi avaliada pelo teste SNK (Sampaio, 2002), utilizando o programa SAEG (Sistema..., 2005). A variável mortalidade embrionária inicial, zero a sete dias de incubação, não teve distribuição de normalidade assim, foi necessário fazer a transformação dos dados, utilizando: raiz (mortalidade inicial $+1 / 2$ ). Portanto, a análise de variância foi feita na função transformada, e as médias comparadas pelo teste SNK. Para as demais idades de mortalidade embrionária, a diferença entre as médias foi avaliada pelo teste Kruskal-Wallis. Estimou-se a correlação de 
Pearson entre o peso do pinto e o peso do ovo e entre o peso do saco vitelino e o peso do pinto.

\section{RESULTADOS E DISCUSSÃO}

Os resultados do experimento I referentes aos pesos absolutos e às variâncias dos pesos dos ovos e das gemas são demonstrados na Tab. 1 .
Com o aumento da idade da matriz, ocorreram aumentos significativos no peso do ovo e no peso da gema. Estes resultados estão de acordo com os encontrados por Peebles et al. (2000); Ferreira et al. (2005); Ribeiro et al. (2007). Isso ocorre porque a produção de gema proveniente da síntese hepática mantém-se a mesma com o avanço da idade da ave, passando a ser depositada em um número menor de folículos, como descrito por Zakaria et al. (1983).

Tabela 1. Média e variância dos pesos dos ovos e das gemas de acordo com a idade das matrizes

\begin{tabular}{|c|c|c|c|c|}
\hline \multirow{2}{*}{ Idade (semanas) } & \multicolumn{2}{|c|}{ Ovo } & \multicolumn{2}{|c|}{ Gema } \\
\hline & Peso (g) & Variância & Peso (g) & Variância \\
\hline 31 & $58,74 \mathrm{c}$ & $16,40 \mathrm{a}$ & $15,30 \mathrm{c}$ & $1,25 \mathrm{a}$ \\
\hline 38 & $66,11 b$ & $17,90 \mathrm{ab}$ & $17,58 \mathrm{~b}$ & $1,54 \mathrm{ab}$ \\
\hline 43 & $66,53 a$ & $21,03 b$ & $19,31 \mathrm{a}$ & $1,93 b$ \\
\hline $\mathrm{CV}(\%)$ & 6,84 & & 6,06 & \\
\hline
\end{tabular}

Médias de pesos seguidas de letras distintas na coluna diferem entre si pelo teste $\mathrm{SNK}(\mathrm{P} \leq 0,05)$.

Variâncias seguidas de letras distintas na coluna indicam valores diferentes pelo teste Fisher $(\mathrm{P} \leq 0,05)$.

A menor variância do peso do ovo e da gema foi observada nos ovos produzidos pelas matrizes com 31 semanas de idade, enquanto as galinhas com 43 semanas de idade produziram ovos e gemas menos uniformes $(\mathrm{P} \leq 0,05)$. Matrizes com 38 semanas de idade apresentaram variância do peso dos ovos e das gemas semelhantes às encontradas nas matrizes com 31 e 43 semanas de idade. Esses resultados diferem dos de Bradalize (2001), que afirmou que lotes de matrizes novas são menos uniformes e, por isso, produzem ovos com pesos mais variados.

Os pesos relativos dos componentes dos ovos do experimento I são apresentados na Tab. 2.

Tabela 2. Porcentagem de gema, albúmen e casca dos ovos de acordo com a idade das matrizes

\begin{tabular}{cccc}
\hline Idade (semanas) & Gema & Albúmen & Casca \\
\hline 31 & $25,75 \mathrm{c}$ & $65,18 \mathrm{a}$ & $9,07 \mathrm{~b}$ \\
38 & $28,00 \mathrm{~b}$ & $62,70 \mathrm{~b}$ & $9,31 \mathrm{a}$ \\
43 & $28,93 \mathrm{a}$ & $61,81 \mathrm{c}$ & $9,26 \mathrm{a}$ \\
CV $(\%)$ & 6,16 & 2,87 & 6,96 \\
\hline
\end{tabular}

Médias seguidas de letras distintas na coluna diferem entre si pelo teste $\mathrm{SNK}(\mathrm{P} \leq 0,01)$.

A porcentagem de gema aumentou e a de albúmen reduziu significativamente com $\mathrm{o}$ aumento da idade da matriz, como anteriormente relatado por Peebles et al. (2000); Ferreira et al. (2005); Joseph e Moran Jr. (2005a); Ribeiro et al. (2007). Das matrizes com 31 semanas resultaram ovos com menor porcentagem de casca que das matrizes com 38 e 43 semanas de idade $(\mathrm{P} \leq 0,01)$.

As taxas de eclosão em relação ao número total de ovos incubados e ao número total de ovos férteis incubados do experimento II são apresentadas na Tab. 3.
Dos ovos do tratamento $\mathrm{F}$ resultou a maior taxa de eclosão sobre o número total de ovos incubados quando comparada aos tratamentos $\mathrm{A}$, $\mathrm{C}$ e $\mathrm{D}(\mathrm{P} \leq 0,05)$. Os ovos dos tratamentos $\mathrm{B}$ e $\mathrm{E}$ tiveram eclosão diferente apenas dos tratamentos A e D. Quando a taxa de eclosão foi calculada em relação ao número de ovos férteis, os ovos do tratamento A resultaram em menor eclodibilidade quando comparada aos demais tratamentos $(\mathrm{P} \leq 0,05)$. As maiores taxas de eclosão foram observadas nos ovos dos tratamentos B, C, E e F. A taxa de eclosão dos ovos do tratamento $\mathrm{D}$ foi intermediária $\mathrm{e}$ semelhante à do tratamento $\mathrm{C}$. 
Efeito da classificação dos ovos...

Tabela 3. Taxa de eclosão em relação ao número total de ovos incubados e ao número de ovos férteis, de acordo com os tratamentos

\begin{tabular}{ccc}
\hline \multirow{2}{*}{ Tratamento } & \multicolumn{2}{c}{ Eclosão (\%) } \\
\cline { 2 - 3 } & Total de ovos incubados & Ovos férteis \\
\hline A & $85,0 \mathrm{c}$ & $89,9 \mathrm{c}$ \\
B & $89,6 \mathrm{ab}$ & $95,2 \mathrm{a}$ \\
$\mathrm{C}$ & $87,1 \mathrm{bc}$ & $93,4 \mathrm{ab}$ \\
$\mathrm{D}$ & $86,4 \mathrm{c}$ & $91,9 \mathrm{~b}$ \\
$\mathrm{E}$ & $89,6 \mathrm{ab}$ & $95,1 \mathrm{a}$ \\
F & $90,4 \mathrm{a}$ & $94,5 \mathrm{a}$ \\
CV $(\%)$ & 3,85 & 2,87 \\
\hline
\end{tabular}

A: ovos com pesos entre 66 e $72 \mathrm{~g}, 33,3 \%$ originados de matrizes com diferentes idades; B: ovos com pesos entre 58 e $65 \mathrm{~g}, 33,3 \%$ originados de matrizes com diferentes idades; C: ovos com pesos entre 52 e $57 \mathrm{~g}$, 33,3\% originados de matrizes com diferentes idades; D: ovos com pesos entre 52 e $72 \mathrm{~g}$, produzidos por matrizes com 31 semanas; E: ovos com pesos entre 52 e $72 \mathrm{~g}$, produzidos por matrizes com 38 semanas; e F: ovos com pesos entre 52 e $72 \mathrm{~g}$, produzidos por matrizes com 43 semanas.

Médias seguidas de letras distintas na coluna diferem entre si pelo teste $\mathrm{SNK}(\mathrm{P} \leq 0,05)$.

A menor eclodibilidade sobre os ovos férteis apresentada pelo tratamento A está de acordo com Wilson (1991), que observou que ovos maiores apresentam baixa eclodibilidade. A baixa taxa de eclosão, verificada no tratamento A, pode ser explicada pela maior mortalidade dos embriões desses ovos a partir de 15 dias de incubação (Tab. 4).
As taxas de mortalidade embrionária inicial, de zero a sete dias, nos demais períodos de incubação - entre oito e 14 dias, entre 15 e 18 dias, entre 19 e 21 dias incluindo os ovos bicados com embriões vivos e mortos, e decorrentes de contaminação ou desidratação dos ovos, observadas no experimento II, são apresentadas na Tab. 4.

Tabela 4. Taxa de mortalidade embrionária, de acordo com os tratamentos e decorrentes de contaminação (CON) ou desidratação (DES)

\begin{tabular}{ccccccc}
\hline Tratamento & $0 \mathrm{a} 7 \operatorname{dias}^{1}$ & $8{\mathrm{a} 14 \operatorname{dias}^{2}}^{15} \mathrm{a} 18$ dias $^{2}$ & Final $^{2,3}$ & $\mathrm{CON}^{2}$ & DES $^{2}$ \\
\hline A & $4,49 \mathrm{bc}$ & 0,13 & $2,60 \mathrm{~b}$ & $4,82 \mathrm{~b}$ & 0,13 & 0,20 \\
B & $3,45 \mathrm{ab}$ & 0,13 & $1,04 \mathrm{a}$ & $2,28 \mathrm{a}$ & 0,20 & 0,13 \\
C & $5,21 \mathrm{c}$ & 0,20 & $0,91 \mathrm{a}$ & $2,60 \mathrm{a}$ & 0,20 & 0,07 \\
D & $4,56 \mathrm{bc}$ & 0,46 & $1,30 \mathrm{a}$ & $3,58 \mathrm{ab}$ & 0,07 & 0,13 \\
E & $4,04 \mathrm{bc}$ & 0,13 & $1,04 \mathrm{a}$ & $2,21 \mathrm{a}$ & 0,46 & 0,20 \\
F & $2,28 \mathrm{a}$ & 0,20 & $1,30 \mathrm{a}$ & $2,54 \mathrm{a}$ & 0,26 & 0,07 \\
\hline
\end{tabular}

A: ovos com pesos entre 66 e $72 \mathrm{~g}, 33,3 \%$ originados de matrizes com diferentes idades; B: ovos com pesos entre 58 e $65 \mathrm{~g}, 33,3 \%$ originados de matrizes com diferentes idades; $\mathrm{C}$ : ovos com pesos entre 52 e $57 \mathrm{~g}, 33,3 \%$ originados de matrizes com diferentes idades; D: ovos com pesos entre 52 e $72 \mathrm{~g}$, produzidos por matrizes com 31 semanas; E: ovos com pesos entre 52 e $72 \mathrm{~g}$, produzidos por matrizes com 38 semanas; e F: ovos com pesos entre 52 e $72 \mathrm{~g}$, produzidos por matrizes com 43 semanas.

${ }^{1}$ Análise de variância feita na variável transformada utilizando raiz (variável + 1/2), porém os dados apresentados na tabela não estão transformados. Médias seguidas de letras distintas na coluna indicam valores diferentes pelo teste $\mathrm{SNK}(\mathrm{P} \leq 0,05) . \mathrm{CV}=22,93 \%$.

${ }^{2}$ Médias seguidas de letras distintas na coluna indicam valores diferentes pelo teste Kruskal-Wallis $(\mathrm{P} \leq 0,05)$.

${ }^{3}$ Final: embriões mortos durante 19 a 21 dias de incubação + embriões bicados vivos e mortos.

A menor taxa de mortalidade embrionária, no período de zero a sete dias de incubação, ocorreu nos ovos do tratamento $\mathrm{F}$ quando comparada aos demais tratamentos $(\mathrm{P} \leq 0,05)$, sendo semelhante somente à taxa de mortalidade inicial apresentada pelo tratamento B. Os ovos do tratamento $\mathrm{C}$ tiveram a maior taxa de mortalidade inicial, porém esta não diferiu das taxas observadas nos tratamentos A, D e E $(\mathrm{P}>0,05)$. 
Não foram observadas diferenças entre os tratamentos quanto à mortalidade embrionária durante o período de oito a 14 dias e decorrente de contaminação ou desidratação dos ovos $(\mathrm{P}>0,05)$.

A partir de 15 dias de incubação, foi observada alta taxa de mortalidade embrionária nos ovos do tratamento A, e esta foi superior aos demais tratamentos $(\mathrm{P} \leq 0,05)$. Essa taxa manteve-se alta até o final do processo de incubação, sendo semelhante estatisticamente apenas aos ovos do tratamento D durante o período final. Essa maior mortalidade dos embriões pode ser explicada pela dificuldade de perda de calor no final do período de incubação, pois o aumento do tamanho do ovo não é acompanhado do aumento proporcional da condutância térmica (French, 1997; Tona et al., 2001; Lourens et al., 2006).

O peso do ovo, do pinto e do saco vitelino, e as relações entre o peso do pinto e o peso do ovo e entre o peso do saco vitelino e o peso do pinto, referentes ao experimento II, são demonstrados na Tab. 5.

Tabela 5. Peso do ovo, do pinto e do saco vitelino e as relações peso do pinto/peso do ovo (PP/PO) e peso do saco vitelino/peso do pinto (PSV/PP), de acordo com os tratamentos

\begin{tabular}{cccccc}
\hline Tratamento & Ovo $(\mathrm{g})$ & Pinto $(\mathrm{g})$ & Saco vitelino $(\mathrm{g})$ & PP/PO $(\%)$ & PSV/PP $(\%)$ \\
\hline A & $68,30 \mathrm{a}$ & $47,03 \mathrm{a}$ & $7,16 \mathrm{a}$ & $68,84 \mathrm{a}$ & $15,15 \mathrm{a}$ \\
B & $60,68 \mathrm{c}$ & $41,87 \mathrm{c}$ & $5,45 \mathrm{~b}$ & $68,99 \mathrm{a}$ & $12,92 \mathrm{~b}$ \\
C & $55,83 \mathrm{e}$ & $37,94 \mathrm{e}$ & $4,30 \mathrm{c}$ & $67,95 \mathrm{a}$ & $11,31 \mathrm{~b}$ \\
D & $58,21 \mathrm{~d}$ & $39,98 \mathrm{~d}$ & $4,80 \mathrm{bc}$ & $68,65 \mathrm{a}$ & $11,92 \mathrm{~b}$ \\
E & $61,66 \mathrm{c}$ & $41,66 \mathrm{c}$ & $5,22 \mathrm{~b}$ & $67,53 \mathrm{a}$ & $12,38 \mathrm{~b}$ \\
F & $65,77 \mathrm{~b}$ & $44,53 \mathrm{~b}$ & $5,59 \mathrm{~b}$ & $67,64 \mathrm{a}$ & $12,47 \mathrm{~b}$ \\
CV $(\%)$ & 5,36 & 7,14 & 27,84 & 3,63 & 23,95
\end{tabular}

A: ovos com pesos entre 66 e 72g, 33,3\% originados de matrizes com diferentes idades; B: ovos com pesos entre 58 e $65 \mathrm{~g}, 33,3 \%$ originados de matrizes com diferentes idades; C: ovos com pesos entre 52 e $57 \mathrm{~g}, 33,3 \%$ originados de matrizes com diferentes idades; D: ovos com pesos entre 52 e $72 \mathrm{~g}$, produzidos por matrizes com 31 semanas; E: ovos com pesos entre 52 e $72 \mathrm{~g}$, produzidos por matrizes com 38 semanas; e F: ovos com pesos entre 52 e $72 \mathrm{~g}$, produzidos por matrizes com 43 semanas.

Médias na coluna seguidas de letras distintas indicam valores diferentes pelo teste $\mathrm{SNK}(\mathrm{P} \leq 0,05)$.

O peso do pinto ao nascer foi proporcional ao peso do ovo, representando, em média, $68,3 \%$ do peso do ovo e sem diferenças entre os tratamentos. Devido à relação proporcional entre os pesos do pinto e do ovo, pode-se observar que o peso do pinto apresentou o mesmo comportamento que o peso do ovo.

Os pesos, absoluto e relativo, do saco vitelino dos pintos provenientes dos ovos do tratamento A foram superiores aos outros tratamentos. Estes resultados estão de acordo com Vieira e Moran Jr. (1998a) e Lourens et al. (2006), sendo que os últimos autores explicaram a superioridade dos pesos absoluto e relativo do saco vitelino pela maior quantidade de nutrientes presente na gema dos ovos mais pesados. Os pintos provenientes dos ovos do tratamento $\mathrm{C}$ apresentaram o menor peso absoluto de saco vitelino, semelhante ao dos oriundos do tratamento D. Os originados dos ovos dos tratamentos B, D, E e F apresentaram pesos absolutos de saco vitelino semelhantes $(\mathrm{P}>0,05)$. Estes resultados não estão de acordo com os de Joseph e Moran Jr. (2005b), porém esses autores avaliaram o peso do saco vitelino um dia após a eclosão e, neste experimento, os sacos vitelinos foram pesados imediatamente após a eclosão. Os pesos relativos dos sacos vitelinos não diferiram entre os pintos provenientes dos ovos dos tratamentos $\mathrm{B}, \mathrm{C}, \mathrm{D}$, E e F. Estes resultados são semelhantes aos encontrados por Vieira e Moran Jr. (1998b; 1999) e contrários aos observados por Latour et al. (1998); Burnham et al. (2001) e Peebles et al. (2001), provavelmente porque esses pesquisadores utilizaram idades de matrizes muito diferentes das utilizadas no presente experimento, além da possível influência do período de avaliação do saco vitelino, nos dois últimos estudos, que foi feita durante o período de incubação.

As correlações de Pearson entre os pesos do pinto e do ovo e os pesos do saco vitelino e do pinto, relativas ao experimento II, são apresentadas na Tab. 6 . 
Tabela 6. Correlação de Pearson para o peso do pinto com o peso do ovo (PP/PO) e o peso do saco vitelino com o peso do pinto (PSV/PP), de acordo com os tratamentos

\begin{tabular}{ccc}
\hline Tratamento & PP/PO & PSV/PP \\
\hline A & 0,61 & 0,64 \\
B & 0,72 & 0,66 \\
C & 0,63 & 0,40 \\
D & 0,94 & 0,68 \\
E & 0,93 & 0,71 \\
F & 0,90 & 0,60 \\
\hline
\end{tabular}

A: ovos com pesos entre 66 e $72 \mathrm{~g}, 33,3 \%$ originados de matrizes com diferentes idades; B: ovos com pesos entre 58 e $65 \mathrm{~g}, 33,3 \%$ originados de matrizes com diferentes idades; C: ovos com pesos entre 52 e $57 \mathrm{~g}, 33,3 \%$ originados de matrizes com diferentes idades; D: ovos com pesos entre 52 e $72 \mathrm{~g}$, produzidos por matrizes com 31 semanas; E: ovos com pesos entre 52 e $72 \mathrm{~g}$, produzidos por matrizes com 38 semanas; e F: ovos com pesos entre 52 e $72 \mathrm{~g}$, produzidos por matrizes com 43 semanas.

Em todos os tratamentos, o peso do pinto foi positivamente correlacionado ao peso do ovo. Estas observações estão de acordo com Wilson (1991). O peso do saco vitelino teve correlação positiva com o peso do pinto, o que está de acordo com os resultados encontrados por Sklan et al. (2003).

\section{CONCLUSÕES}

Matrizes jovens produzem ovos e gemas com pesos mais uniformes do que matrizes mais velhas. Embriões de ovos mais pesados apresentam maior mortalidade a partir de 15 dias de incubação, resultando em menor taxa de eclosão. O peso do saco vitelino é correlacionado ao peso do pinto, e o peso do pinto é correlacionado ao peso do ovo.

\section{REFERÊNCIAS BIBLIOGRÁFICAS}

BENTON, C.E.; BRAKE, J. The effect of broiler breeder age and lenght of egg storage on egg albumen during early incubation. Poult. Sci., v.75, p.1069-1075, 1996.

BRADALIZE, V.H. A influência da nutrição da matriz sobre o desempenho do frango de corte. In: ENCONTRO TÉCNICO DE CIÊNCIAS AVIÁRIAS, 5., 2001, Uberlândia. Anais... Uberlândia: UFU, 2001. p.42-71.
BRAKE, J.; WALSH, T.J.; BENTON, C.E. et al. Egg handling and storage. Poult. Sci., v.76, p.144-151, 1997.

BURNHAM, M.R.; PEEBLES, E.D.; GARDNER, C.W. et al. Effects of incubator humidity and hen age on yolk composition in broiler hatching eggs from young breeders. Poult. Sci., v.80, p.1444-1450, 2001.

FASENKO, G.M. Candling and hatch residue breakouts. In: ROBINSON, F.E.; FASENKO, G.M.; RENEMA, R.A. (Eds). Optimizing chick production in broiler breeders. Canada: Spotted Cow, 2003. p.101-104.

FERREIRA, F.C.; LARA, L.J.C.; BAIÃO, N.C. et al. Influência da idade da matriz sobre a qualidade do ovo. Rev. Bras. Cien. Avic., v.7, supl., p.16, 2005.

FIÚZA, M.A.; LARA, L.J.C.; AGUILAR, C.A.L. et al. Efeitos das condições ambientais no período entre a postura e o armazenamento de ovos de matrizes pesadas sobre o rendimento de incubação. Arq. Bras. Med. Vet. Zootec., v.58, p.408-413, 2006.

FRENCH, N.A. Modeling incubation temperature: the effects of incubator design, embryonic development, and egg size. Poult. Sci., v.76, p.124-133, 1997.

JOSEPH, N.S.; MORAN JR., E.T. Characteristics of eggs, embryos, and chicks from broiler breeder hens selected for growth or meat yield. J. Appl. Poult. Res., v.14, p.275-280, 2005a.

JOSEPH, N.S.; MORAN JR., E.T. Effect of flock age and postemergent holdin in the hatcher on broiler live performance and furtherprocessing yield. J. Appl. Poult. Res., v.14, p.512-520, 2005b.

LATOUR, M.A.; PEEBLES, E.D.; DOYLE, S.M. et al. Broiler breeder age and dietary fat influence the yolk fatty acid profiles of fresh eggs and newly hatched chicks. Poult. Sci., v.77, p.47-53, 1998 .

LOURENS, A.; MOLENAAR, R.; VAN DEN BRAND, H. et al. Effect of egg size on heat production and the transition of energy from egg to hatchling. Poult. Sci., v.85, p.770-776, 2006.

MARINHO, J.C.; LARA, L.J.C.; BAIÃO, N.C. et al. Efeitos da idade da matriz e do peso do ovo sobre as relações entre peso do pinto e peso do 
saco vitelino. Rev. Bras. Cien. Avic., v.8 supl., p.22, 2006.

MICHALSKY, V.B.; CANÇADO, S.V.; LARA, L.J.C. et al. Influência da umidade na incubação e idade da matriz leve sobre a eclosão e parâmetros de ovos e pintos. Rev. Bras. Cien. Avic., v.7, supl. p.13, 2005.

PAPPAS, A.C.; ACAMOVIC, N.H.C.; SPARKS, N.H.C. et al. Effects of suplementing broiler breeder diets with organoselenium compounds and polyunsatured fatty acids on hatchability. Poult. Sci., v.85, p.1584-1593, 2006.

PEEBLES, E.D.; DOYLE, S.M.; ZUMWALT, C.D. et al. Breeder age influences embyogenesis in broiler hatching eggs. Poult. Sci., v.80, p.272$277,2001$.

PEEBLES, E.D.; ZUMWALT, C.D.; DOYLE, S.M.; GERARD, P.D. et al. Effects of breeder age and dietary fat source and level on broiler hatching egg characteristics. Poult. Sci., v.79, p.698-704, 2000.

RIBEIRO, B.R.C.; LARA, L.J.C.; BAIÃO, N.C. et al. Efeito do nível de ácido linoléico na ração de matrizes pesadas sobre o peso, composição e eclosão dos ovos. Arq. Bras. Med. Vet. Zootec., v.59, p.789-796, 2007.

SAMPAIO, I.B.M. Estatística aplicada à experimentação animal. 2.ed. Belo Horizonte: FEPMVZ, 2002, 265p.
SISTEMA de análises estatísticas - SAEG. Versão 9.0. Viçosa: UFV, 2005.

SKLAN, D.; HEIFETZ, S.; HALEVY, O. Heavier chicks at hatch improves marketing body weight by enhancing skeletal muscle growth. Poult. Sci., v.82, p.1778-1786, 2003.

TONA, K.; BAMELIS, F.; COUCKE, W. et al. Relantionship between broiler breeder's age and egg weight loss and embryonic mortality during incubation in large-scale conditions. J. Appl. Poult. Res., v.10, p.221-227, 2001.

VIEIRA, S.L.; MORAN JR., E.T. Broiler chicks hatched from egg weight extremes and diverse breeder strains. J. Appl. Poult. Res., v.7, p.392402, 1998a.

VIEIRA, S.L.; MORAN JR., E.T. Broiler yields using chicks from egg weight extremes and diverse strains. J. Appl. Poult. Res., v.7, p.339346, 1998 b.

VIEIRA, S.L.; MORAN JR., E.T. Effects of egg of origin and chick post-hatch nutrition on broiler live performance and meat yields. World's Poult. Sci. J., v.55, p.125-142, 1999.

WILSON, H.R. Interrelationships of egg size, chick size, posthatching growth and hatchability. World's Poult. Sci. J., v.47, p.5-20, 1991.

ZAKARIA, A.H.; MIYAKI, T.; IMAI, K. The effect of aging on the ovarian folicular growth in laying hens. Poult. Sci., v.62, p.670-674, 1983. 